\title{
The infrared properties of the new outburst star IRAS 05436-0007 in quiescent phase
}

\author{
P. Ábrahám ${ }^{1}$, Á. Kóspál ${ }^{2}$, Sz. Csizmadia ${ }^{1}$, A. Moór ${ }^{1}$, M. Kun ${ }^{1}$, and G. Stringfellow ${ }^{3}$ \\ ${ }^{1}$ Konkoly Observatory of the Hungarian Academy of Sciences, PO Box 67, 1525 Budapest, Hungary \\ 2 Department of Astronomy, Eötvös Loránd University, PO Box 32, 1518 Budapest, Hungary \\ ${ }^{3}$ Center for Astrophysics \& Space Astronomy, University of Colorado at Boulder, 389 UCB, Boulder, CO 80309-0389, USA
}

Received 12 March 2004 / Accepted 10 April 2004

\begin{abstract}
We compiled and investigated the infrared/sub-mm/mm SED of the new outburst star IRAS 05436-0007 in quiescent phase. The star is a flat-spectrum source, with an estimated total luminosity of $L_{\mathrm{bol}} \approx 5.6 L_{\odot}$, typical of low-mass T Tauri stars. The derived circumstellar mass of $0.5 M_{\odot}$ is rather high among low-mass YSOs. The observed SED differs from the SEDs of typical T Tauri stars and of 4 well-known EXors, and resembles more the SEDs of FU Orionis objects indicating the presence of a circumstellar envelope. IRAS $05436-0007$ seems to be a class II source with an age of approximately $4 \times 10^{5} \mathrm{yr}$. In this evolutionary stage an accretion disk is already fully developed, though a circumstellar envelope may also be present. Observations of the present outburst will provide additional knowledge on the source.
\end{abstract}

Key words. stars: formation - stars: circumstellar matter - stars: individual: IRAS 05436-0007 - infrared: stars

\section{Introduction}

On 23 Jan. 2004 the amateur astronomer McNeil discovered a new nebula towards the Orion B molecular cloud, close to the diffuse nebulosity Messier 78 (McNeil et al. 2004). The object was not visible in either of the two Palomar Surveys (1951, 1990), but a photograph taken in 1966 for the book "The Messier Album"1 (Mallas \& Kreimer 1978) shows a bright nebulosity very similar to the one of today. Also in the very deep [SII] image of Eislöffel \& Mundt (1997), taken in October 1995, parts of the nebula are clearly visible though fainter than in 1966. The alternation of active and quiescent periods, suggested by these earlier observations, indicates that the event, probably the eruption of a pre-main sequence star, may be similar to the well-known EXor-type outbursts.

At infrared and sub-millimetre wavelengths, however, the source was observable also during the quiescent periods (IRAS, 2MASS, Lis et al. 1999; Mitchell et al. 2001). At these wavelengths the emission is due to thermal radiation of circumstellar dust. The infrared/sub- $\mathrm{mm} / \mathrm{mm}$ data offer a possibility to study the circumstellar matter - which is likely responsible for the explosion via a sudden rise of the accretion onto the star (Hartmann \& Kenyon 1996) - prior to an outburst.

In this paper we collect all infrared/sub-mm observations available in the literature and compile a spectral energy distribution (SED) representative of the quiescent phase. The SED

Send offprint requests to: P. Ábrahám, e-mail: abraham@konkoly .hu ${ }^{1}$ http://www . seds.org/messier/ will be analysed, and compared with SEDs of pre-main sequence stars, including several known FUORs and EXors.

\section{Infrared/sub-mm/mm data}

From the literature one can collect several infrared and sub-mm/mm flux values for the new outburst star:

The 2MASS All-Sky Catalog of Point Sources (Cutri et al. 2003) contains a source coinciding with the optical position of the new star. We adopt the coordinates of this source, J05461313-0006048, as the position of the outburst object: $\alpha_{2000}=5^{\mathrm{h}} 46^{\mathrm{m}} 13^{\mathrm{s}} .13, \delta_{2000}=-0^{\circ} 6^{\prime} 4^{\prime \prime} \cdot 8$. Table 1 presents the 2MASS $J H K_{\mathrm{S}}$ fluxes, which are all flagged as high quality data in the catalogue.

In the ISO Data Archive ${ }^{2}$ there are two mid-infrared maps, taken by the ISOCAM instrument, which covers the position of the star. Checking the source lists available in the FITS file headers, it was possible to identify a point source coinciding with the 2MASS source. Its flux densities (produced by ISO's automatic off-line processing software) are also given in Table 1.

The outburst star was identified with the IRAS source 05436-0007 by Eislöffel \& Mundt (1997). We adopted the 12 and $25 \mu \mathrm{m}$ flux densities from the IRAS Point Source Catalogue (Table 1). At longer wavelengths the PSC gives only upper limit, therefore we determined new IRAS fluxes using the SCANPI Processing Tool at IPAC ${ }^{3}$. At $60 \mu \mathrm{m}$ it was

\footnotetext{
${ }^{2}$ http://www.iso.vilspa.esa.es/IDA

${ }^{3}$ http://irsa.ipac.caltech.edu/applications/Scanpi
} 
Table 1. Infrared/sub-mm/mm observations of IRAS 05436-0007 collected from the literature (Sect. 2). No reddening correction was applied. We adopt the coordinates of the 2MASS source, $\alpha_{2000}=$ $5^{\mathrm{h}} 46^{\mathrm{m}} 13.13 \delta_{2000}=-0^{\circ} 6^{\prime} 4^{\prime \prime} 8$, as the position of the outburst star.

\begin{tabular}{cccl}
\hline \hline $\begin{array}{c}\lambda \\
\mu \mathrm{m}\end{array}$ & $\begin{array}{c}\text { Flux } \\
{[\mathrm{mJy}]}\end{array}$ & $\begin{array}{c}\text { Obs. } \\
\text { date }\end{array}$ & Name, reference \\
\hline 1.25 & 1.997 & 1998 & 2MASS J05461313-0006048 \\
1.65 & 13.94 & 1998 & 2MASS J05461313-0006048 \\
2.17 & 50.96 & 1998 & 2MASS J05461313-0006048 \\
\hline 6.7 & 267 & 1997 & ISOCAM map, ISO_id: 68901103 \\
14.3 & 559 & 1997 & ISOCAM map, ISO_id: 68901122 \\
\hline 12 & 527 & 1983 & IRAS 05436-0007, PSC \\
25 & 1200 & 1983 & IRAS 05436-0007, PSC \\
60 & 2000 & 1983 & IRAS 05436-0007, Scanpi \\
\hline 350 & 2500 & 1997 & LMZ 12, Lis et al. (1999) \\
850 & 180 & 1998 & Ori B smm 55, Mitchell et al. (2001) \\
1300 & 93 & 1998 & LMZ 12, Lis et al. (1999) \\
\hline
\end{tabular}

possible to extract an estimated $2 \pm 1 \mathrm{Jy}$, but at $100 \mu \mathrm{m}$ no meaningful flux or upper limit could be determined due to the complex background of the Orion B molecular cloud. In the remaining of this paper we use the IRAS name when referring to the new outburst star.

Three sub-mm/mm measurements can be assigned to IRAS 05436-0007 at 350 and $1300 \mu \mathrm{m}$ (Lis et al. 1999) and at $850 \mu \mathrm{m}$ (Mitchell et al. 2001), taking into account the spatial resolutions of the used instruments. At $1300 \mu \mathrm{m}$ the source was unresolved, while at $850 \mu \mathrm{m}$ a $\sim 20^{\prime \prime}$ object was visible. The total integrated fluxes, taken from the papers, as well as the names the authors gave to the object are given in Table 1.

\section{Quiescent periods of the source}

IRAS 05436-0007 was not visible in either of the two Palomar Surveys $(1951,1990)$, showing that the source was in quiescence. In order to define better the active and quiescent periods of the star, we checked the photographic archive of the Konkoly Observatory (containing more than 13000 Schmidt plates) and discovered two - a blue sensitive and an approximately visual photographs for the area of the outburst star. From a visual inspection of the plates we concluded that the source was not visible either in 1964 October or in 1976 October (the estimated detection limit was $17.5 \pm 0.5 \mathrm{mag}$ in both plates). Thus the eruption recorded in 1966 on the photograph in "The Messier Album" (Sect. 1) was not long-lasting. From the listed data one may probably conclude that IRAS 05436-0007 was mainly in quiescent phase throughout the second half of the last century.

\section{The infrared SED}

In Fig. 1 we plotted all data points listed in Table 1. It is not obvious - due to their different epochs - if they form a physically consistent SED representative of the quiescent phase. In the near-infrared regime, however, the 2MASS images do not reveal any extended nebulosity suggesting that the source was

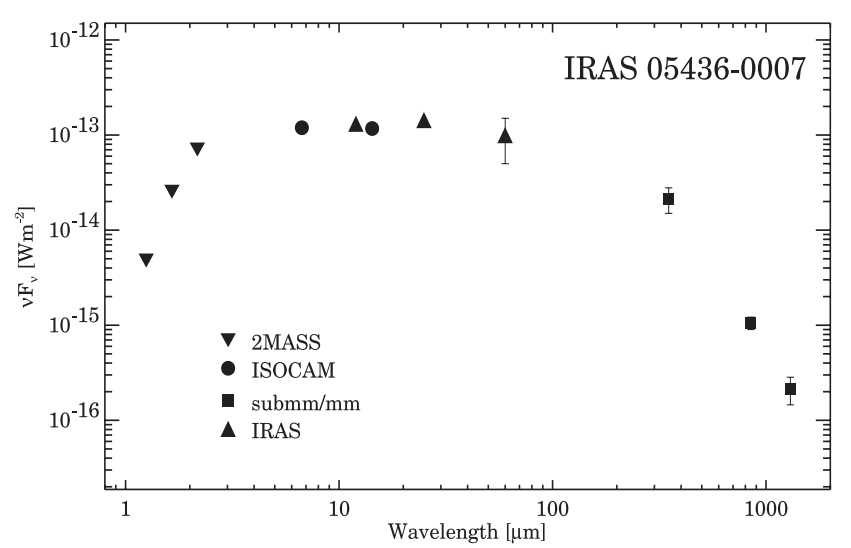

Fig. 1. Spectral energy distributions of IRAS 05436-0007. The fluxes are taken from the literature and are listed in Table 1.

inactive at that date. In the mid-infrared there exist observations of two different epochs (IRAS, ISOCAM), whose good agreement suggests a similar activity level - probably quiescence at the two epochs. At far-infrared wavelengths our study of 7 FU Orionis-type stars (Kóspál et al. 2004) suggests that the flux densities of eruptive stars are practically independent of the outburst stage, and probably the same is true for the sub$\mathrm{mm} / \mathrm{mm}$ regime. Thus combining our multiepoch data into a single SED seems to be justified. In the following we discuss the different wavelength regimes of this SED separately.

Near-infrared: the unusually high $J-H$ and $H-K$ indices indicate that the central source is heavily extincted. Figure 2 shows the location of the star on a $J-H$ vs. $H-K$ diagram. A backward projection parallel to the reddening path onto the locus of the classical T Tauri stars (Meyer et al. 1997) gives dereddened colour indices of $(J-H)_{0}=1.15 \mathrm{mag}$ and $(H-K)_{0}=1.08 \mathrm{mag}$. The near-infrared reddening is $E(J-H)=1.43 \mathrm{mag}$, and using the relationship of Rieke \& Lebofsky (1985) this result gives a visual interstellar extinction of $A_{\mathrm{V}} \approx 13 \mathrm{mag}$. One should keep in mind that the observed near-infrared colours could be affected by scattering within the circumstellar environment, therefore the derived extinction value (and also the result of the reddening correction in Fig. 3) should be taken with some caution. The $(J-H)_{0}$ and $(H-K)_{0}$ values indicate the presence of a significant amount of circumstellar matter.

Mid-infrared (7-25 $\mu \mathrm{m})$ : based on the IRAS and sub-mm data points, Lis et al. (1999, their Fig. 6) modelled the SED as a sum of two modified Planck functions with $T=165 \mathrm{~K}$ and $T=30 \mathrm{~K}$. The inclusion of the ISOCAM fluxes, however, does not support this simple model, but indicates that IRAS 05436-0007 is a flat-spectrum source at infrared wavelengths. Such a SED requires a less steep radial temperature profile than expected from an optically thick, geometrically thin circumstellar disk, and can be the result either of a flared disk (Adams et al. 1988) or of an extended envelope. Detailed modelling of FU Ori-type objects (Turner et al. 1997) showed that flared disks alone may not be able to produce a flat spectrum over an extended wavelength range, thus in many cases 


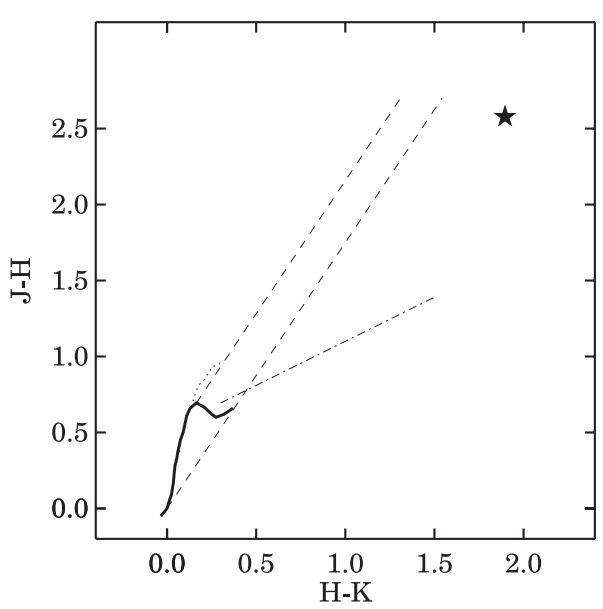

Fig. 2. The location of IRAS 05436-0007 (star) on a near-infrared colour-colour diagram. Overplotted are the unreddened main sequence (solid line) and giant sequence (dotted line), the reddening path of the main sequence (dashed line), and also the locus of the dereddened classical T Tauri stars (dashed-dot line, Meyer et al. 1997).

envelopes are invoked to explain the SEDs of the flat-spectrum sources in the infrared.

Far-infrared: the flat spectrum declines at longer wavelengths. This part of the SED was fitted by Lis et al. (1999) assuming a modified Planck-function $B_{v}(T) \nu^{\beta}$ with $T \sim 30 \mathrm{~K}$ and $\beta \sim 1.0$. Including the $850 \mu \mathrm{m}$ data point of Mitchell et al. (2001) does not change the fit. The turning point in the spectral shape, which marks the transition between optically thick and thin emission, is probably between 100 and $200 \mu \mathrm{m}$. Since in many classical $\mathrm{T}$ Tauri stars the turning point is in the range of 60 to $100 \mu \mathrm{m}$ (e.g. D'Alessio et al. 1999), the fit results indicate the presence of a large amount of cold material in the outer part of the IRAS 05436-0007 system. The submillimetre spectral slope, characterised by $\beta \sim 1$, shows that the average particle size is larger than the typical value in the interstellar medium $(\beta \sim 2)$, probably due to grain evolution in the circumstellar environment.

In order to compute the luminosity of IRAS 05436-0007, we applied a reddening correction on the SED of Fig. 1 assuming $A_{\mathrm{V}}=13 \mathrm{mag}$. The result is shown in Fig. 3. Integrating over the corrected SED between 1 and $1300 \mu \mathrm{m}$ gives a total infrared-to-submillimetre luminosity of $5.6 L_{\odot}$ (for the distance of the Ori B cloud $d=460$ pc was adopted). This value is higher than the estimate of Lis et al. $\left(1999, L_{\mathrm{bol}} \sim\right.$ $2.7 L_{\odot}$ ) but the discrepancy is explained by the inclusion of the new near-infrared data points and by the reddening correction. The obtained luminosity of $5.6 L_{\odot}$ clearly shows that IRAS 05436-0007 is a low-mass T Tauri-like object.

From the sub-mm flux densities it is possible to estimate the circumstellar mass. Lis et al. (1999) computed 0.4 $M_{\odot}$, while from the measurement of Mitchell et al. (2001, Eq. (4)) we derived $\sim 0.6 M_{\odot}$. The obtained mass is rather high compared to typical T Tau values of $\sim 0.01 M_{\odot}$ (e.g. Beckwith et al. 1990).

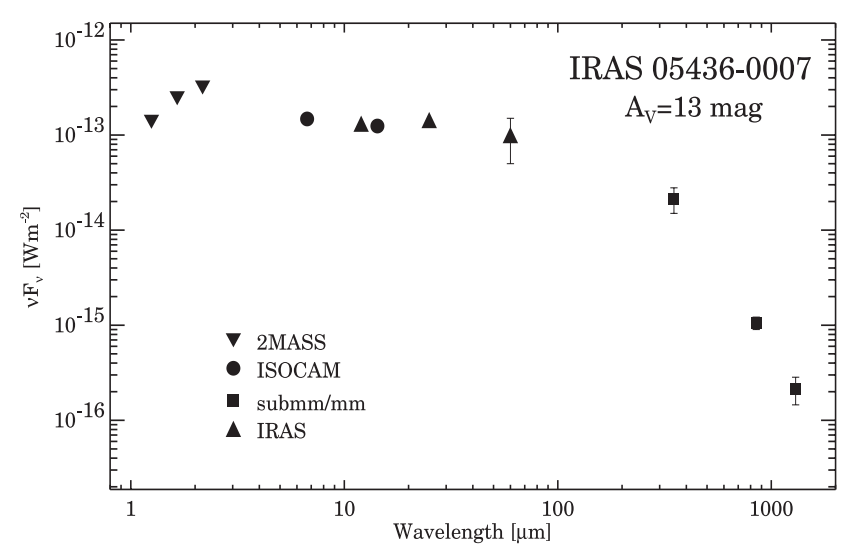

Fig. 3. Spectral energy distributions of IRAS 05436-0007 after correcting for an interstellar extinction of $A_{\mathrm{V}}=13 \mathrm{mag}$.

\section{Discussion}

\subsection{Comparison with pre-main sequence stars}

In this section we compare the SED of IRAS 05436-0007 with a sample of SEDs of pre-main sequence stars. We focus on the $\lambda \geq 10 \mu \mathrm{m}$ spectral range, because at optical/near-infrared wavelengths the variation of circumstellar extinction with inclination angle introduces a diversity in the spectral shapes.

T Tauri stars. The median SED of 39 young stars from the Taurus-Auriga star-forming region was constructed by D'Alessio et al. (1999). Their Fig. 5 reveals that the median SED declines towards longer wavelengths in the $10-100 \mu \mathrm{m}$ range, which is clearly inconsistent with the flat spectrum of IRAS 05436-0007.

EXors. The infrared SEDs of 4 well-known EXor-type objects (EX Lup, DR Tau, UZ Tau, VY Tau) in quiescent phase were derived from ISOPHOT observations by Stringfellow et al. (2004). Similarly to the T Tauri stars, these objects also exhibit SEDs declining towards longer wavelengths, significantly differing from the SED of IRAS 05436-0007. On the other hand, the intermediate-mass young star PV Cephei, which is sometimes classified as an eruptive EXor-like variable (e.g. Teodorani et al. 1999) shows an approximately flat SED (Ábrahám et al. 2000).

FUORs. In Fig. 4 we collected the infrared SEDs of 6 FU Ori-type objects published in Kóspál et al. (2004), which are in the post-outburst phase. All these SEDs are flat $\left(v F_{v} \sim\right.$ const.) or even raising with increasing wavelength in the $10-100 \mu \mathrm{m}$ spectral range. These SEDs resemble more the SED of the new outburst star (Fig. 1) than do either the SEDs of the T Tau-stars or the EXors. Especially the 3 sources with flat mid-infrared spectra: V346 Nor, V1057 Cyg, and ZCMa, look similar to IRAS 05436-0007 (though in the case of $\mathrm{ZCMa}$ the companion, a Herbig Ae/Be star, might also contribute to the SED). 

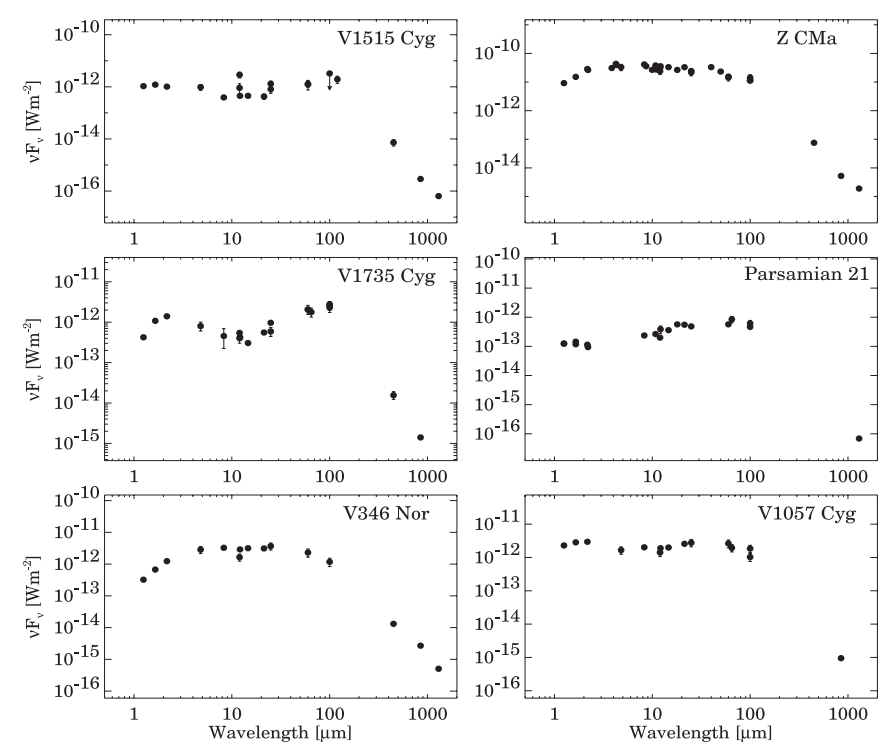

Fig. 4. Spectral energy distributions of 6 FUORs (from Kóspál et al. 2004). The presented data are based on observations obtained by ISOPHOT, the photometer on-board the Infrared Space Observatory, supplemented by IRAS, MSX, 2MASS, and sub-mm/mm data. In case the SED changed between 1983 and 1997 (Kóspál et al. 2004) only the more recent data set was plotted.

The fact that IRAS 05436-0007 was bright also in 1966 (Sect. 3) suggests multiple active periods, i.e. an EXor-like nature. Its luminosity of $5.6 L_{\odot}$ (Sect. 4 ) is also more typical for the T Tau-like EXors than for the 5-100 times more luminuous FUORs (Sandell \& Weintraub 2001). However, on the basis of the shape of the spectral energy distribution, IRAS 05436-0007 is more similar to the FU Orionis objects. The flat spectra of the FUORs are usually interpreted in terms of extended circumstellar envelopes (Kenyon \& Hartmann 1991; Turner et al. 1997), whose material is falling onto the outer parts of the accretion disk. Thus IRAS 05436-0007 might also be surrounded by an envelope, which - together with the unusually large circumstellar mass of $\approx 0.5 M_{\odot}$ (Sect. 4) - would make this object relatively unique among the known EXors. Observations of the present outburst, including measurements of the infrared SED with the Spitzer Space Telescope, will provide more data to compute detailed models of the circumstellar structure.

\subsection{Evolutionary stage of IRAS 05436-0007}

Based on the submillimetre-to-bolometric luminosity ratio Lis et al. (1999) proposed that IRAS 05436-0007 is a relatively young and embedded Class 0 source, though some observations (e.g. the lack of molecular outflow) seemed to indicate that the source was more evolved. In order to estimate the evolutionary stage of the source, we followed the method of Chen et al. (1995) and computed the bolometric temperature $T_{\text {bol }}$ according to their Eq. (1). The resulting $T_{\text {bol }}=830 \mathrm{~K}$ and the bolometric luminosity $L_{\text {bol }} \approx 5.6 L_{\odot}$ was then compared with the distribution of corresponding values among YSOs in the Taurus and $\rho$ Oph star forming regions (Chen et al. 1995). From this check we can conclude that IRAS 05436-0007 seems to be a Class II object (close to the Class I/Class II boundary), and its age - according to Fig. 4 of Chen et al. (1995) - is approximately $4 \times 10^{5} \mathrm{yr}$.

\section{Conclusions}

We compiled and investigated the infrared/sub-mm/mm SED of the new outburst star IRAS 05436-0007 in quiescent phase. The star is a flat-spectrum source, with an estimated total luminosity of $L_{\mathrm{bol}} \approx 5.6 L_{\odot}$, typical of low-mass T Tauri stars. The derived circumstellar mass of $0.5 M_{\odot}$ is rather high among low-mass YSOs. The observed SED differs from the SEDs of typical T Tauri stars and of 4 well-known EXors, and resembles more the SEDs of FU Orionis objects indicating the presence of a circumstellar envelope. IRAS 05436-0007 seems to be a Class II source with an age of approximately $4 \times 10^{5} \mathrm{yr}$. In this evolutionary stage an accretion disk is already fully developed, though a circumstellar envelope may also be present. Observations of the present outburst will provide additional knowledge on the source.

Acknowledgements. We thank an anonymous referee whose comments improved the manuscript significantly. The work was supported by the grants OTKA T 037508 and T 034584 of the Hungarian Scientific Research Fund. P.Á. acknowledges the support of the Bolyai Fellowship.

\section{References}

Ábrahám, P., Leinert, Ch., Burkert, A., Henning, Th., \& Lemke, D. 2000, A\&A, 354, 965

Adams, F. C., Lada, C. J., \& Shu, F. H. 1988, ApJ, 326, 865

Beckwith, S. V. W., Sargent, A. I., Chini, R. S., \& Guesten, R. 1990, AJ, 99, 924

Chen, H., Myers, P. C., Ladd, E. F., \& Wood, D. O. S. 1995, ApJ, 445, 377

Cutri, R. M., Skrutskie, M. F., Van Dyk, S., et al. 2003, Explanatory Supplement to the 2MASS All Sky Data Release (Pasadena: Caltech)

D’Alessio, P., Calvet, N., Hartmann, L., Lizano, S., \& Cantó, J. 1999, ApJ, 527, 893

Eislöffel, J., \& Mundt, R. 1997, AJ, 114, 280

Hartmann, L., \& Kenyon, S. J. 1996, ARA\&A, 34, 207

Lis, D. C., Menten, K. M., \& Zylka, R. 1999, ApJ, 527, 856

Kenyon, S. J., \& Hartmann, L. 1991, ApJ, 383, 664

Kóspál, Á., Ábrahám, P., \& Csizmadia, Sz. 2004, Baltic Astron., 12, in press [arXiv: astro-ph/0402292]

Mallas, J. H., \& Kreimer, E. 1978, The Messier Album (Sky Publishing Corporation)

McNeil, J. W., Reipurth, B., \& Meech, K. 2004, IAUC 8284, 1

Meyer, M. R., Calvet, N., \& Hillenbrand, L. A. 1997, AJ, 114, 288

Mitchell, G. F., Johnstone, D., Moriarty-Schieven, G., Fich, M., \& Tothill, N. F. H. 2001, ApJ, 556, 512

Rieke, G. H., \& Lebofsky, M. J. 1985, ApJ, 288, 618

Sandell, G., \& Weintraub, D. A. 2001, ApJS, 134, 115

Stringfellow, G., et al. 2004, in preparation

Teodorani, M., Errico, L., \& Vittone, A. A. 1999, Mem. Soc. Astron. Ital., 70, 417

Turner, N. J. J., Bodenheimer, P., \& Bell, K. R. 1997, ApJ, 480, 754 